\title{
Ocorrência de carrapatos em Tamandua tetradactyla (Linnaeus, 1758) na Caatinga, Nordeste do Brasil
}

\author{
[Occurrence of ticks in Tamandua tetradactyla (Linnaeus, 1758) in Caatinga biome, northeastern Brazil] \\ G.M.B. Oliveira ${ }^{1}$, T.F. Martins ${ }^{2}$, L.C.M. Pereira ${ }^{1}$, P.A. Nicola ${ }^{1}$, M.C. Horta ${ }^{1 *}$ \\ 1Universidade Federal do Vale do São Francisco - Petrolina, PE \\ ${ }^{2}$ Universidade de São Paulo - São Paulo, SP
}

\section{RESUMO}

\begin{abstract}
A Caatinga é um bioma exclusivamente brasileiro e um dos mais diversificados do mundo, abrigando grande variedade faunística. Este estudo objetivou relatar a presença de carrapatos em tamanduás Tamandua tetradactyla (Linnaeus, 1758) atendidos no Centro de Conservação e Manejo de Fauna da Caatinga, PetrolinaPE, Brasil. De fevereiro de 2010 a fevereiro de 2016, 23 T. tetradactyla foram recebidos e inspecionados para avaliação da presença de carrapatos. A identificação foi realizada pela observação das características morfológicas e pela utilização de chaves taxonômicas. Aproximadamente 8,69\% (2/23) dos animais apresentavam parasitismo por carrapatos. Os ixodídeos foram encontrados fixados nas regiões ventral e cervical, e identificados como Rhipicephalus sanguineus sensu lato (cinco fêmeas), Amblyomma auricularium (uma larva, 18 ninfas e uma fêmea) e Amblyomma sp. (seis larvas). Este estudo relata o parasitismo inédito de larva de A. auricularium em $T$. tetradactyla no semiárido pernambucano, bem como reafirma a maior afinidade por membros da superordem Xenarthra. O parasitismo de $R$. sanguineus s.l. em tamanduá-mirim pode estar relacionado ao compartilhamento do mesmo habitat com cães domésticos infestados. $\mathrm{O}$ parasitismo sobre animais silvestres em novas localidades onde não foi registrado previamente deve ser estudado para ampliação do conhecimento sobre a distribuição geográfica dos ixodídeos no Brasil.
\end{abstract}

Palavras-chave: tamanduá-mirim, Amblyomma auricularium, Rhipicephalus sanguineus sensu lato, Caatinga, semiárido

\begin{abstract}
The Caatinga is an exclusively Brazilian biome and one of the most diverse in the world, housing a great variety faunal. Southern Tamandua (Linnaeus, 1758), known as collared anteater, can be found throughout the country. The present study aimed to report the presence of ticks on anteaters treated at the Conservation Center and Wildlife Management of Caatinga, Petrolina, Pernambuco, Brazil. From February 2010 to February 2016, 23 T. tetradactyla from the municipalities of Juazeiro (BA) Casa Nova (BA), Sento Sé (BA), Nonato (PI), Petrolina (PE), Great Pond (PE) and Willow (PE), have been received and inspected to evaluate the presence of ticks, which were later collected with the help of anatomical tweezer and stored in tubes containing $70 \%$ alcohol. The identification was carried out by observation of morphological characteristics in stereomicroscope and use of taxonomic keys. Approximately $8.69 \%(2 / 23)$ of the animals had parasitism by ticks. The Ixodids were found fixed on ventral and cervical regions and were identified as Rhipicephalus sanguineus sensu lato (five female), parasitizing the anteater 1 coming from Petrolina-PE; Amblyomma auricularium (one larva, 18 nymphs and a female) and Amblyomma sp. parasitizing the anteater 2 coming from Salgueiro-PE. This study reports the novel parasitism by larva of A. auricularium in T. tetradactyla in semiarid of Pernambuco ; and reaffirms the higher affinity of this ixodid by members of the superorder Xenarthra. It was possible to determine the parasitism of R. sanguineus s.l. in collared anteater, the which may be related to the sharing of the same habitat with domestic dogs infested. The parasitism of wild animals in new locations where they were not previously registered must be stimulated to expand the knowledge of the geographical distribution of ixodids in Brazil.
\end{abstract}

Keywords: Collared anteater, Amblyomma auricularium, Rhipicephalus sanguineus sensu lato, Caatinga, semiarid

Recebido em 24 de agosto de 2016

Aceito em 10 de outubro de 2016

*Autor para correspondência (corresponding author)

E-mail: horta.mc@hotmail.com 


\section{INTRODUÇÃO}

A Caatinga é um bioma exclusivamente brasileiro e um dos mais diversificados do mundo (Tabarelli e Silva, 2003), abrigando uma grande variedade faunística, incluindo cerca de 156 espécies de mamíferos, dentre eles o Tamandua tetradactyla (Linnaeus, 1758), popularmente conhecido como "tamanduámirim" ou "tamanduá-de-colete" (Albuquerque et al., 2012).

A espécie T. tetradactyla é um mamífero de médio porte, com pelagem curta e densa, e apresenta duas listras pretas, que avançam da região escapular até a porção posterior do animal, semelhantemente a um colete. Com hábito predominantemente noturno e escansorial, utiliza tocas de tatu, ocos de árvores e outras cavidades naturais para descansar nos períodos de inatividade. Essa espécie apresenta ampla distribuição territorial, sendo encontrada ao longo da América do Sul, da Venezuela ao norte da Argentina, a leste da Cordilheira dos Andes, norte do Uruguai e no Brasil,onde está presente em todos os biomas. A alimentação desse mamífero é constituída basicamente de formigas, cupins, mel e abelhas, extraídos com suas garras dianteiras, por meio da ruptura dos ninhos desses insetos (Medri et al., 2011).

Assim como a maioria das espécies de mamíferos, $T$. tetradactyla está sujeito a infestações por ectoparasitos, sendo comumente parasitado por carrapatos do gênero Amblyomma (Martins et al., 2004). Entre as espécies desse gênero, Amblyomma auricularium (Conil, 1878), popularmente conhecido como "carrapatovermelho-do-tatu", já foi registrado parasitando diversas espécies de animais silvestres no bioma Caatinga (Horta et al., 2011).

Os centros de conservação atuam com programas de resgate e monitoramento de fauna silvestre, auxiliando na geração de conhecimento sobre as diferentes espécies animais, os parasitas e o meio ambiente em que vivem. Nesse contexto, o presente estudo objetivou relatar a presença de carrapatos em tamanduás-mirim atendidos no Centro de Conservação e Manejo de Fauna da Caatinga, localizado no município de PetrolinaPE, Brasil.

\section{MATERIAL E MÉTODOS}

No período de fevereiro de 2010 a fevereiro de 2016, 23 tamanduás-mirim foram resgatados e encaminhados ao Centro de Conservação e Manejo de Fauna da Caatinga (Cemafauna), localizado na Universidade Federal do Vale do São Francisco, em Petrolina-PE, sendo seis oriundos do Ibama, em Juazeiro-BA, quatro sob os cuidados do mesmo órgão federal, com sede em Salgueiro-PE, um resgatado pelo Corpo de Bombeiros, em Petrolina-PE, dois resgatados em áreas de instalação de Parques Eólicos, na Bahia, um resgatado pela Secretaria de Meio Ambiente de Sento-Sé (BA), um resgatado nas áreas de instalação do Projeto de Integração do Rio São Francisco, em Pernambuco, um encaminhado pelo ICMBio de São Raimundo Nonato (PI), quatro oriundos do Inema de Juazeiro (BA) e, por fim, uma entrega voluntária por particulares dos municípios de Petrolina, Salgueiro e Casa Nova. Os municípios estão localizados no semiárido do nordeste brasileiro, que apresenta uma paisagem típica desse clima (IBGE, 2005).

Durante o exame físico realizado nos animais no momento da entrada no Centro, foram coletados carrapatos em fase de parasitismo, fixados na região ventral e cervical. Os ectoparasitas foram retirados com pinça anatômica e, posteriormente, armazenados em recipiente plástico contendo um fragmento de gramínea, visando manter a umidade do microambiente. As formas imaturas foram mantidas em estufa, para promoção da ecdise (Horta et al., 2011). A identificação dos espécimes foi realizada com auxílio de estereomicroscópio e de chaves taxonômicas, segundo Aragão e Fonseca (1961), BarrosBattesti et al. (2006) e Martins et al. (2010).

\section{RESULTADOS}

Dos 23 animais avaliados, dois (8,69\%) apresentavam parasitismo por carrapatos. No primeiro animal (resgatado pelo Corpo de Bombeiros, em Petrolina-PE), foram coletadas cinco fêmeas, identificadas como Rhipicephalus sanguineus sensu lato (Latreille, 1806). No segundo animal (oriundo do Ibama, em Salgueiro-PE), foram coletados 26 carrapatos: sete larvas identificadas como Amblyomma sp., tendo uma delas sofrido ecdise na estufa para uma ninfa que posteriormente foi identificada como A. auricularium; 18 ninfas identificadas 
como A. auricularium, das quais 17 sofreram ecdise para o estágio adulto (oito machos e nove fêmeas); e uma fêmea de A. auricularium.

\section{DISCUSSÃO}

As espécies de Amblyomma relatadas em $T$. tetradactyla de vida livre e mantidos em cativeiro no Brasil foram: Amblyomma aureolatum, Amblyomma auricularium, Amblyomma brasiliense, Amblyomma cajennense, Amblyomma calcaratum, Amblyomma dubitatum, Amblyomma goeldii, Amblyomma humerale, Amblyomma nodosum, Amblyomma parvum e Amblyomma sculptum (citado como Amblyomma cajennense) (Martins et al., 2004, Arzua et al., 2005, Quadros et al., 2009, Dantas-Torres et al., 2010a, Dantas-Torres et al., 2010b, Lavina et al., 2011, Spolidorio et al., 2012, Garcia et al., 2013, Martins et al., 2014, 2015a,b, 2016, Santos et al., 2015, Soares et al., 2015, Bastos et al., 2016, Witter et al., 2016).

A menor abundância de espécies do gênero Amblyomma no presente estudo apresenta uma correlação com as características do bioma Caatinga, que possui condições mais hostis a esses artrópodes, principalmente no que diz respeito às altas temperaturas e à baixa umidade (Horta et al., 2011). O parasitismo por outras espécies desse gênero, como A. dubitatum e A. nodosum, já foi relatado em área de Mata Atlântica, no Nordeste do Brasil (Dantas-Torres et al., 2010a,b).

O carrapato A. auricularium apresenta ampla distribuição no continente americano, sendo descrito em diversos países, como: Argentina, EUA, Bolívia, Brasil, Colômbia, Costa Rica, Guiana Francesa, Honduras, México, Nicarágua, Panamá, Paraguai, Trinidad e Tobago, Uruguai e Venezuela (Guglielmone et al., 2003). É comumente encontrado em tatus, como tatugalinha (Dasypus novemcinctus), tatu-bola (Tolypeutes matacus), tatu-de-quinze-quilos (Dasypus kappleri), tatu-peba (Euphractus sexcinctus), e numa variedade de pequenos mamíferos, como cangambá (Conepatus semistriatus), preá (Galea spixii), cuíca (Monodelphis domestica), cuíca-verdadeira (Philander opossum), rato-rabudo (Thrichomys apereoides), entre outros (Guglielmone et al., 2003; Barros-Battesti et al., 2006; Horta et al., 2011).
Possivelmente o parasitismo de A. auricularium em tamanduá-mirim esteja relacionado ao hábito desse mamífero de descansar em cavidades naturais, como tocas de tatus (Medri et al., 2011; Desbiez e Kluyber, 2013), uma vez que, segundo Guglielmone et al. (2003), os tatus são frequentemente encontrados parasitados por esse ixodídeo, e Hoogstraal e Aeschlimann (1982) apontam essa espécie como um carrapato com moderada especificidade por mamíferos da família Dasypodidae.

Embora existam relatos de A. auricularium em estágios adulto (Guglielmone et al., 2003) e de ninfa (Dantas-Torres et al. 2010a) parasitando $T$. tetradactyla, o presente estudo relata, de forma inédita, pela primeira vez, a presença de larvas de A. auricularium parasitando T. tetradactyla no bioma Caatinga. Esse fato reforça a necessidade de estudos mais aprofundados sobre a relação do parasitismo de A. auricularium em tamanduás e em outras espécies silvestres, para um maior conhecimento de seus hospedeiros naturais para todos os estágios, principalmente imaturos.

Neste estudo, foi registrado o parasitismo por $R$. sanguineus s.l. em T. tetradactyla, reforçando, dessa forma, o achado prévio desse ixodídeo em tamanduá-mirim por Teixeira e Miranda (2012), contudo essa relação tem maior influência da manutenção de animais silvestres em cativeiro (Labruna et al., 2005). Sugere-se que a ocorrência de $R$. sanguineus s.l. em tamanduámirim pode estar relacionada ao compartilhamento do mesmo habitat com cães domésticos infestados pela mesma espécie de carrapato, uma vez que os caninos são considerados os únicos hospedeiros primários dessa espécie (Labruna e Pereira, 2001).

\section{CONCLUSÃO}

O presente trabalho relata a ocorrência de $A$. auricularium e $R$. sanguineus s.1. em $T$. tetradactyla nativos da região semiárida e encaminhados ao Centro de Conservação e Manejo de Fauna da Caatinga e contribui para o conhecimento sobre o parasitismo nesses animais, destacando a necessidade de novos estudos que colaborem para $o$ melhor entendimento das inter-relações entre hospedeiro, parasita e meio ambiente em que vivem. 


\section{AGRADECIMENTOS}

Ao Conselho Nacional de Pesquisa(CNPq) e à Fundação de Amparo à Ciência e Tecnologia do Estado de Pernambuco (Facepe), pelo apoio financeiro.

\section{REFERÊNCIAS}

ALBUQUERQUE, U.P.; ARAÚJO, E.L.; ELDEIR, A.C.A. et al. Caatinga revisited: ecology and conservation of an important seasonal Dry Forest. Sci. World J., v.2012, p.1-18, 2012.

ARAGÃO, H.B.; FONSECA, F. Notas de ixodologia. VIII: Lista e chave para os representantes da fauna ixodológica brasileira. Mem. Inst. Oswaldo Cruz., v.59, p.115-129, 1961.

ARZUA, M.; ONOFRIO, V.C.; BARROSBATTESTI, D.M. Catalogue of the tick collection (Acari: Ixodidae) of the Museu de História Natural Capão da Imbuia, Curitiba, Paraná, Brazil. Rev. Bras. Zool., v.22, p.623-632, 2005.

BARROS-BATTESTI, D.M.; ARZUA, M.; BECHARA, G.H. Carrapatos de importância médico-veterinária da região Neotropical: um guia ilustrado para identificação de espécies. São Paulo: Vox, 2006. 223p.

BASTOS, T.S.A.; MADRID, D.M.C.; FARIA, A.M. et al. Carrapatos em animais silvestres do bioma cerrado triados pelo cetas, IBAMA-Goiás. Ciênc. Anim. Bras., v.17, p.296-302, 2016.

DANTAS-TORRES, F.; FERREIRA, D.R.; MELO, L.M. et al. Ticks on captive and freeliving wild animals in northeastern Brazil. Exp. Appl. Acarol., v.50, p.181-189, 2010b.

DANTAS-TORRES, F.; SIQUEIRA, D.B.; RAMEH-DE-ALBUQUERQUE, L.C. et al. Ticks infesting wildlife species in northeastern Brazil with new host and locality records. $J$. Med. Entomol., v.47, p.1243-1246, 2010a.

DESBIEZ, A.L.J.; KLUYBER, D. The role of giant armadillos (Priodontes maximus) as physical ecosystem engineers. Biotropica, v.45, p.537-540, 2013.
GARCIA, M.V.; SILVA, D.C.; ALMEIDA, R.F.C. et al. Environmentally associated ticks (Acari: Ixodidae) in Campo Grande, Mato Grosso do Sul, Brazil. Rev. Bras. Parasitol. Vet., v.22, p.124-128, 2013.

GUGLIELMONE, A.A.; ESTRADA-PEÑA, A.; LUCIANI, C.A. et al. Hosts and distribution of Amblyomma auricularium (Conil 1878) and Amblyomma pseudoconcolor Aragão, 1908 (Acari: Ixodidae). Exp. Appl. Acarol., v.29, p.131-139, 2003.

HOOGSTRAAL, H.; AESCHLIMANN, A. Tick-host specificity. Mitt. Schweiz. Entomol. Ges., v.55, p.5-32, 1982.

HORTA, M.C.; NASCIMENTO, G.F.; MARTINS, T.F. et al. Ticks (Acari: Ixodida) parasitizing free-living wild animals in the caatinga biome in the state of Pernambuco, northeastern Brazil. Syst. Appl. Acarol., v.16, p.207-211, 2011.

IBGE. CADASTRO DE MUNICÍPIOS LOCALIZADOS NA REGIÃO SEMIÁRIDA DO BRASIL. [Rio de Janeiro]: IBGE, [2005]. Disponível em: <http://www.ibge.gov.br/home/geociencias/geog rafia/semiarido.shtm?c=4>. Acessado em: 07 mai. 2016.

LABRUNA, M.B.; JORGE, R.S.P.; SANA, D.A. et al. Ticks (Acari: Ixodida) on wild carnivores in Brazil. Exp. Appl. Acarol., v.36, p.149-163, 2005.

LABRUNA, M.B.; PEREIRA, M.C. Carrapato em cães no Brasil. Clín. Vet., v.30, p.24-32, 2001.

LAVINA, M.S.; QUADROS, R.M.; CARNEIRO JÚNIOR, J.A. et al. Primeiro registro de Amblyomma calcaratum (Acari: Ixodidae) em tamanduá-mirim (Tamandua tetradactyla) no Planalto Catarinense, Brasil. Vet. Foco., v.9, p.32-35, 2011.

MARTINS, J.R.; MEDRI, I.M.; OLIVEIRA, C.M.; GUGLIELMONE, A. Ocorrência de carrapatos em tamanduá-bandeira (Myrmecophaga tridactyla) e tamanduá-mirim (Tamandua tetradactyla) na região do Pantanal Sul Mato-grossense, Brasil. Cienc. Rural, v.34, p.293-295, 2004. 
MARTINS, T.F.; BARBIERI, A.R.; COSTA, F.B. et al. Geographical distribution of Amblyomma cajennense (sensu lato) ticks (Parasitiformes: Ixodidae) in Brazil, with description of the nymph of A. cajennense (sensu stricto). Parasit. Vectors, v.9, p.186, 2016.

MARTINS, T.F.; GIANIZELLA, S.L.; NUNES, P.H. et al. New records of Amblyomma goeldii (Acari: Ixodidae) and description of the nymphal stage. Zootaxa, v.3949, p.439-444, 2015a.

MARTINS, T.F.; ONOFRIO, V.C.; BARROSBATTESTI, D.M.; LABRUNA, M.B. Nymphs of the genus Amblyomma (Acari: Ixodidae) of Brazil: descriptions, redescriptions, and identification key. Ticks Tick Borne Dis., v.1, p.75-99, 2010.

MARTINS, T.F.; TEIXEIRA, R.H.F.; LABRUNA, M.B. Ocorrência de carrapatos em animais silvestres recebidos e atendidos pelo Parque Zoológico Municipal Quinzinho de Barros, Sorocaba, São Paulo, Brasil. Braz. J. Vet. Res. Anim. Sci., v.52, p.319-324, 2015b.

MARTINS, T.F.; VENZAL, J.M.; TERASSINI, F.A. et al. New tick records from the state of Rondônia, western Amazon, Brazil. Exp. Appl. Acarol., v.62, p.121-128, 2014.

MEDRI, I.M.; MOURÃO, G.M.; RODRIGUES, F.H.G. Ordem Pilosa. In: REIS, N.R.; PERACCHI, A.L.; PEDRO, W.A.; LIMA, I.P. (Eds.). Mamíferos do Brasil. Londrina: Nelio R. dos Reis, 2011. 439p.

QUADROS, R.M.; BENEDET, R.; MAZZOLLI, M.; MARQUES, S.M.T. Amblyomma nodosum (acari: ixodidae) infesting collared anteater (Tamandua tetradactyla, linnaeus, 1758) in Urupema, Santa Catarina, Brazil. Biociências, v.17, p.73-84, 2009.
SANTOS, P.M.S.; SILVA, S.G.N.; FONSECA, C.F.; OLIVEIRA, J.B. Parasitos de aves e mamíferos silvestres em cativeiro no estado de Pernambuco. Pesqui. Vet. Bras., v.35, p.788-794, 2015.

SOARES, H.S.; BARBIERI, A.R.M.; MARTINS, T.F. et al. Ticks and rickettsial infection in the wildlife of two regions of the Brazilian Amazon. Exp. Appl. Acarol., v.65, p.125-140, 2015.

SPOLIDORIO, M.G.; ANDREOLI, G.S.; MARTINS, T.F. et al. Rickettsial infection in ticks collected from road-killed wild animals in Rio de Janeiro, Brazil. J. Med. Entomol., v.49, p.1510-1514, 2012.

TABARELLI, M.; SILVA, J.M.C. Áreas e ações prioritárias para a conservação da biodiversidade da Caatinga. In: LEAL, I.; SILVA, J.M.C.; TABARELLI, M. (Eds.). Ecologia $e$ conservação da Caatinga. Recife: UFPE, 2003. p.781-800.

TEIXEIRA, R.H.F.; MIRANDA, F. Ixodofauna em tamanduás. In: MIRANDA, F. (Ed.). Manutenção de tamanduás em cativeiro. São Carlos: Instituto de Pesquisa e Conservação de Tamanduás no Brasil: Projeto Tamanduá: Cubo, 2012. p.256-261.

WITTER, R.; MARTINS, T.F.; CAMPOS A.K. et al. Rickettsial infection in ticks (Acari: Ixodidae) of wild animals in midwestern Brazil. Ticks Tick Borne Dis., v.7, p.415-423, 2016. 